\title{
Absolute zero in Casimir vacuum
}

\section{Xingwu Xu}

Research Institute of Hefei Gotion High-tech Power Energy Co, Ltd

599 Daihe Road, Hefei, Anhui 230012, P. R. China

E-mail addresses: xuxingwu@gotion.com.cn

Received Dec 2019

Received in revised: Feb 2020

Published: March 2020

\begin{abstract}
Casimir vacuum has many peculiar properties, e.g. the speed of light can exceed c. This paper investigated the behavior of gas molecules in Casimir vacuum. Analysis and calculation indicate that we can get a new absolute zero in Casimir vacuum. This is due to the Hamiltonian, which equals the kinetic energy of the ensemble of gas molecules that is reduced in Casimir vacuum; therefore, the gas constant $\mathrm{R}$ is changed. This conclusion has great significance for the research of the vacuum properties.
\end{abstract}

Keywords: Casimir vacuum, Absolute zero

C2020 The Authors. Published by Fundamental Journals. This is an open access article under the CC BY-NC https://creativecommons.org/licenses/by-nc/4.0/

https://doi.org/10.14331/ijfps.2020.330133

\section{INTRODUCTION}

Casimir vacuum has many peculiar properties. For example, Scharnhorst found that the speed $c^{\perp}$ of light normal to the Casimir parallel mirrors could exceed $c=1$ (Barton, 1990; Scharnhorst, 1990). In fact, the vacuum structure has been modified in Casimir vacuum. The propagation of light depends on the structure of the vacuum (Scharnhorst, 1990). The main factor is that the refractive index (n) in Casimir vacuum is changed,

$$
n(0)=1-k \rho \quad k=\frac{44 \alpha^{2}}{135 m^{4}}
$$

Where $\rho$ is the vacuum energy density, $\alpha$ is the fine structure constant, $\mathrm{m}$ is the electron mass. The results of Scharnhost's calculation are,

$$
\begin{gathered}
n_{\|}(0)=1 \\
\mathrm{n}_{\perp}(0)=1-\frac{11 \pi^{2}}{(90)^{2}} \frac{\alpha^{2}}{(\mathrm{~mL})^{4}}
\end{gathered}
$$

Where $L$ is the distance of two plates. Therefore, $n_{\perp}(0)<1$, and the propagation velocity $\left(\mathrm{c} / \mathrm{n}_{\perp}\right)$ of light perpendicular to the mirrors will be faster than $\mathrm{c}$. This conclusion does not contradict the special theory of relativity (Scharnhorst, 1998). This research stimulated a broader interest in the study of the properties of vacuum. The question raised in this paper is: Will Casimir vacuum affect the value of absolute zero?

https://doi.org/10.14331/ijfps.2020.330133

ISSN: 2231-8186/ @2020 The Authors. Published by Fundamental Journals

This is an open access article under the CC BY-NC @ (1) (8)

https://creativecommons.org/licenses/by-nc/4.0/ 


\section{THE INTERACTION BETWEEN MOLECULES OR ATOMS AND THE VACUUM FIELD}

This issue is related to the reaction between molecules or atoms of gas and the vacuum field. The molecules or atoms moving in the ordinary vacuum will change their momentum due to the reaction between molecules or atoms and the vacuum. Bercegol and Lehoucq (2015) state that there is a braking torque in a rotating pair of atoms when reacting with quantum vacuum, which decreases the kinetic energy and the angular momentum of the atoms. Recently, Sonnleitner found that an excited two-level atom moving through a vacuum sees a tiny friction force (Sonnleitner, Trautmann, \& Barnett, 2017). The friction force arises from the interaction between atoms and the vacuum field. The interaction can be described by the so-called "Rontgen term $\left(\mathrm{H}_{\mathrm{AF}}\right)$ " (Wilkens, 1994).

$$
H_{A F}=-\hat{\mathbf{d}} \cdot \widehat{\boldsymbol{E}}^{\perp}(\widehat{\boldsymbol{R}})-\frac{1}{2 \mathrm{M}}\{\widehat{\boldsymbol{P}} \cdot[\widehat{\boldsymbol{B}}(\widehat{\boldsymbol{R}}) \times \widehat{\boldsymbol{d}}]+[\widehat{\boldsymbol{B}}(\widehat{\boldsymbol{R}}) \times \widehat{\boldsymbol{d}}] \cdot \widehat{\boldsymbol{P}}\}
$$

Here $\widehat{\boldsymbol{d}}$ is the atomic dipole operator, $\widehat{\boldsymbol{P}}$ and $\widehat{\boldsymbol{R}}$ are the atomic center-of-mass canonical momentum and position operator, $\widehat{\boldsymbol{E}}^{\perp}$ is the electric field, and $\widehat{\boldsymbol{B}}$ is the magnetic field:

$$
\begin{gathered}
\widehat{\boldsymbol{E}}^{\perp}(\widehat{\boldsymbol{R}})=\mathrm{i} \sum_{\mathrm{k}, \lambda} \varepsilon_{\mathrm{k}} \mathbf{e}_{\mathbf{k}, \lambda}\left(\hat{\mathrm{a}}_{\mathrm{k}, \lambda} \mathrm{e}^{\mathrm{ik} \cdot \widehat{\mathbf{R}}}-\hat{\mathrm{a}}_{\mathrm{k}, \lambda}^{\dagger} \mathrm{e}^{-\mathrm{ik} \cdot \widehat{\mathbf{R}}}\right) \\
\widehat{\boldsymbol{B}}(\widehat{\boldsymbol{R}})=\frac{\mathrm{i}}{\mathrm{c}} \sum_{\mathrm{k}, \lambda} \varepsilon_{\mathrm{k}}\left(\boldsymbol{n}_{k} \times \mathbf{e}_{\mathrm{k}, \lambda}\right)\left(\hat{\mathrm{a}}_{\mathrm{k}, \lambda} \mathrm{e}^{\mathrm{i} \mathbf{k} \cdot \widehat{\mathbf{R}}}-\hat{\mathrm{a}}_{\mathrm{k}, \lambda}^{\dagger} \mathrm{e}^{-\mathrm{i} \mathbf{k} \cdot \widehat{\mathbf{R}}}\right)
\end{gathered}
$$

Where $\varepsilon_{\mathrm{k}}=\sqrt{\hbar \omega_{\mathrm{k}} /\left(2 \omega_{0} \mathrm{~V}\right)}$ is the electric field strength per photon in quantization volume $V, \boldsymbol{n}_{k}=\boldsymbol{k} /|\boldsymbol{k}|$ is a unit vector in the direction of propagation, $\boldsymbol{e}_{k, \lambda}$ is a polarization vector perpendicular to the mode propagating in a direction $\boldsymbol{K}=$ $\mathbf{k c} / \omega_{\mathrm{k}}$ (Sonnleitner et al., 2017), $\hat{a}_{k, \lambda}$ and $\hat{a}_{k, \lambda}^{\dagger}$ denote the photon annihilation and creator operators of the $k^{t h}$ mode with the polarization $\lambda(\lambda=1,2)$. In fact, the "Rontgen term" is a negative energy dissipation term caused by the displacement current. The interaction mentioned above results in the decrease of the momentum of atoms. Because the kinetic momentum $p=M v$ ( $M$ : mass, $v$ : velocity), the change of momentum will be (Sonnleitner et al., 2017).

$$
\dot{p}=M \dot{v}+\dot{M} v
$$

The conclusion is that the velocities of atoms do not change; the atom's decay in momentum comes from the mass defect caused by the photon's emission.

\section{THE KINETIC ENERGY OF GAS MOLECULES IN CASIMIR VACUUM}

However, if we put the gas molecules in the Casimir vacuum, the kinetic energy will change. For a two-level atom, the sum of the center-of-mass kinetic energy is (Wilkens, 1994).

$$
\mathrm{H}=\frac{\widehat{\mathbf{P}}^{2}}{2 \mathrm{M}}+H_{A}+H_{F}+H_{A F}
$$

Where $H_{A}$ is the atomic Hamiltonian, $H_{F}$ is the free field Hamiltonian, and $H_{A F}$ is the atom-field interaction Hamiltonian. Now, we consider an ensemble of gas molecules in Casimir vacuum. The total kinetic energy of gas molecules is,

$$
E_{\text {kin-sys }}=\sum H
$$

In fact, the Casimir vacuum is a space where some $\mathrm{k}$ modes (longer wavelength) are excluded, as shown in Fig. 1 (Chown, 1990).

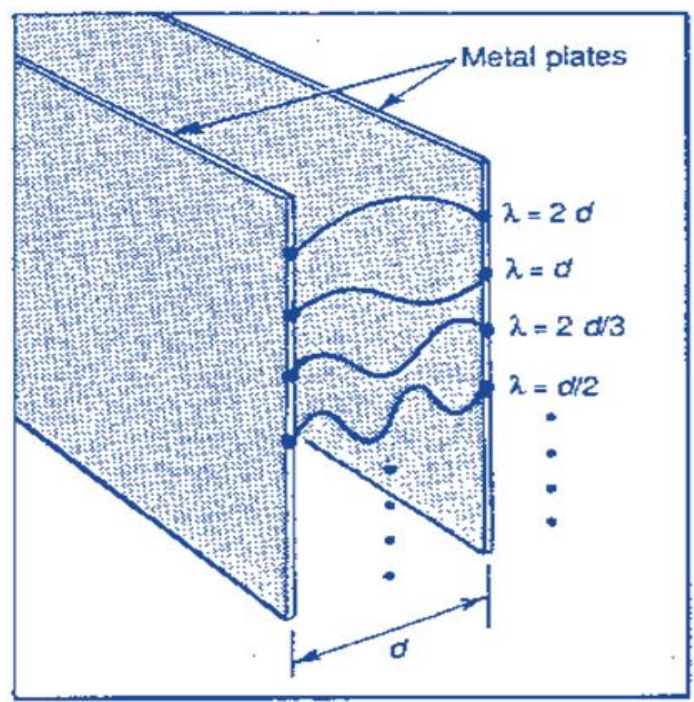

Fig 1. Casimir effect: The vacuum is full of virtual photons, but photons with wavelength $(\lambda)$ more than twice the separation of the plates are excluded from the space between them.

The Rontgen term is actually the friction term with negative value. For the sum operation, if the value of term is positive, the greater the number of terms, the greater the sum. Conversely, if the value of term is negative, the larger the number of terms, the smaller the sum. Now let us use $k^{\prime \prime}$ for the number of modes (terms) in Casimir vacuum, and $k^{\prime}$ for the number of modes in the ordinary vacuum. From equations (4) $\sim(6)$, we know that for $k^{\prime \prime}<k^{\prime}$ (Fig. 1), we have

$$
H_{A F}^{\prime \prime}>H_{A F}^{\prime}
$$

Equation (10) indicates that in Casimir vacuum, the mean coupling energy between the ensemble of gas molecules and the field is lower than in ordinary vacuum. Therefore, we have

$$
E_{\text {kin-ens }}^{\prime \prime}<E_{\text {kin-ens }}^{\prime}
$$

Where $E_{\text {kin-ens }}$ denotes the kinetic energy of the ensemble of gas molecules. From another viewpoint, one can divide the kinetic energy of the gas ensemble into two parts-the selfenergy of the gas molecules and the energy of the vacuum field. A coupling exists between the gas molecules and the vacuum field; the total energy of gas molecules is the coupling effect of two parts of energies. Thus,

$$
E_{\text {kin-sys }}=E_{\text {self }}+E_{\text {vac }}
$$


From (Bercegol \& Lehoucq, 2015) we know that,

$$
E_{v a c}=-\frac{\pi^{2}}{720 L^{3}}
$$

Where $L$ is the distance between two plates, $h=c=1$. Therefore, the kinetic energy of gas molecules in Casimir vacuum is lower than that in an ordinary vacuum

$$
E_{\text {kin-ens }}^{\prime \prime}\left(=E_{\text {self }}-\frac{\pi^{2}}{720 \mathrm{~L}^{3}}\right)<E_{\text {kin-ens }}^{\prime}\left(=E_{\text {self }}\right)
$$

\section{A NEW ABSOLUTE ZERO IN CASIMIR VACUUM}

According to (Fischer et al., 2007), temperature is proportional to the mean translational kinetic energy of atoms or molecules; it is just the well-known equation

$$
\bar{E}_{k i n}=\frac{3}{2} k_{B} \mathrm{~T}
$$

Where $\bar{E}_{k i n}$ can be considered same as $E_{\text {kin-ens }}$. Now, the paradox arises: in Casimir vacuum at absolute zero temperature, $E^{\prime \prime}{ }_{k i n-e n s}<{E^{\prime}}_{k i n-e n s}$, which term will change$\mathrm{k}_{\mathrm{B}}$ or $\mathrm{T}$ ? We have two options: 1) $\mathrm{T}$ decreases or 2) $\mathrm{k}_{\mathrm{B}}$ decreases.

1) If we put the Casimir vacuum as well as the ensemble of gas molecules into absolute zero, the temperature in Casimir vacuum will be less than absolute zero,

$$
T_{\text {Casi }}<0 \mathrm{~K}
$$

2) If the $\mathrm{k}_{\mathrm{B}}$ decreases, according to the relation:

$$
k_{B}=\frac{R}{N_{A}}
$$

Where $R$ is the gas constant, $N_{A}$ is the Avogadro constant, then $\mathrm{R}$ will decrease. Back to the gas law,

$$
\frac{P_{0} V_{0}}{T_{0}}=R
$$

Where 0 here denotes the standard condition, $P_{0}=1.01325 \times$ $10^{5} P_{a}$ is the pressure, $V_{0}=22.414 \times 10^{-3} \mathrm{~m}^{3}$ is the volume of gas, $T_{0}=273.15 \mathrm{~K}$ is absolute temperature, and $R=$ 8.314. If $R$ changes, then $T_{0}$ will change. In standard conditions, the average kinetic energy for a single molecule is,

$$
\bar{E}_{\text {kin }}^{\prime}=\frac{3}{2} k_{B} T_{0}=5.656864 \times 10^{-21} \mathrm{~J}
$$

Suppose the distance between two conductive plates is $0.1 \mu$, the size of the plate is $1 \mathrm{~m} \times 1 \mathrm{~m}$; then, the mole number of the gas in Casimir vacuum is

$$
n=\frac{V_{\text {Casi }}}{V_{0}}=4.4615 \times 10^{-6}
$$

The total average kinetic energy of gas molecules in Casimir vacuum is

$$
\bar{E}_{k i n}=\bar{E}_{k i n}^{\prime} n N_{A}=1.519838 \times 10^{-2} J
$$

The negative vacuum energy is

$$
E_{v a c}=-\frac{\mathrm{hc \pi} \pi^{2}}{720 \mathrm{~L}^{3}}=-2.75 \times 10^{-7} \mathrm{~J}
$$

In Casimir vacuum, the total average kinetic energy of gas molecules becomes

$$
\bar{E}_{\text {kin }}^{\text {Casi }}=\bar{E}_{k i n}+E_{\text {vac }}=1.5198105 \times 10^{-2} J
$$

The average kinetic energy for a single molecule in Casimir vacuum is

$$
\bar{E}_{k i n}^{\prime \prime}=\frac{\bar{E}_{\text {kin }}^{\text {Casi }}}{n N_{A}}=5.655928 \times 10^{-21} \mathrm{~J}
$$

Therefore, the new $k_{B}$ is

$$
k_{B}^{\text {Casi }}=\frac{2}{3} \frac{\bar{E}_{\text {kin }}^{\prime \prime}}{T_{0}}=1.38042 \times 10^{-23}
$$

The new gas constant is

$$
R_{\text {Casi }}=k_{B}^{\text {Casi }} N_{A}=8.31289
$$

The absolute zero in Casimir vacuum is

$$
\begin{gathered}
T_{\text {Casi }}=-\frac{V_{0} P_{0}}{R_{\text {Casi }}}=-273.20 \mathrm{~K} \\
\Delta \mathrm{T}=T_{\text {Casi }}-T_{K}=-273.20-(-273.15)=-0.05 \mathrm{~K}
\end{gathered}
$$

The conclusion shows that the change of vacuum structure will result in a new absolute zero temperature. It is possible that in another planet, where the vacuum structure is different from earth, there will be a new absolute zero, which will obey the third law of thermodynamics there.

\section{PROOF AND MEASUREMENT}

As a "thought experiment", the new absolute zero can be proved and measured by designing a design of Casimir vacuum pump (Fig. 2). As illustrated in Fig.2, the "modern Magdeburg hemispheres" consist of several hollow spheres. There are many Casimir slits in the shells. In order to shield the photons or other field particles with longer wavelengths, these slits can be designed in staggered patterns, which are in twists and turns. Moreover, these shells have to have enough strength to bear the huge pressure. Moreover, the shell material itself also has a weak shielding function. The next step is to "pump" out these photons as well as field particles with longer wavelengths in the hollow space. Two Casimir plates are installed in this hollow space. Both the plates have one of their ends separated by a small distance (Casimir slit), and the other end can open or close. When opening, there are many photons as well as other field particles in the containing space. Then, the two plates move together, leaving a Casimir slit between them. Meanwhile, the doors in the shells close synchronously. By repeating this operation, the larger space with Casimir vacuum can be obtained. When opening, there are many photons as well as other field particles in the containing space. Then, the two plates move together, leaving a Casimir slit between them. Meanwhile, the doors in the shells close synchronously. By repeating this operation, the larger space with Casimir vacuum can be obtained. 


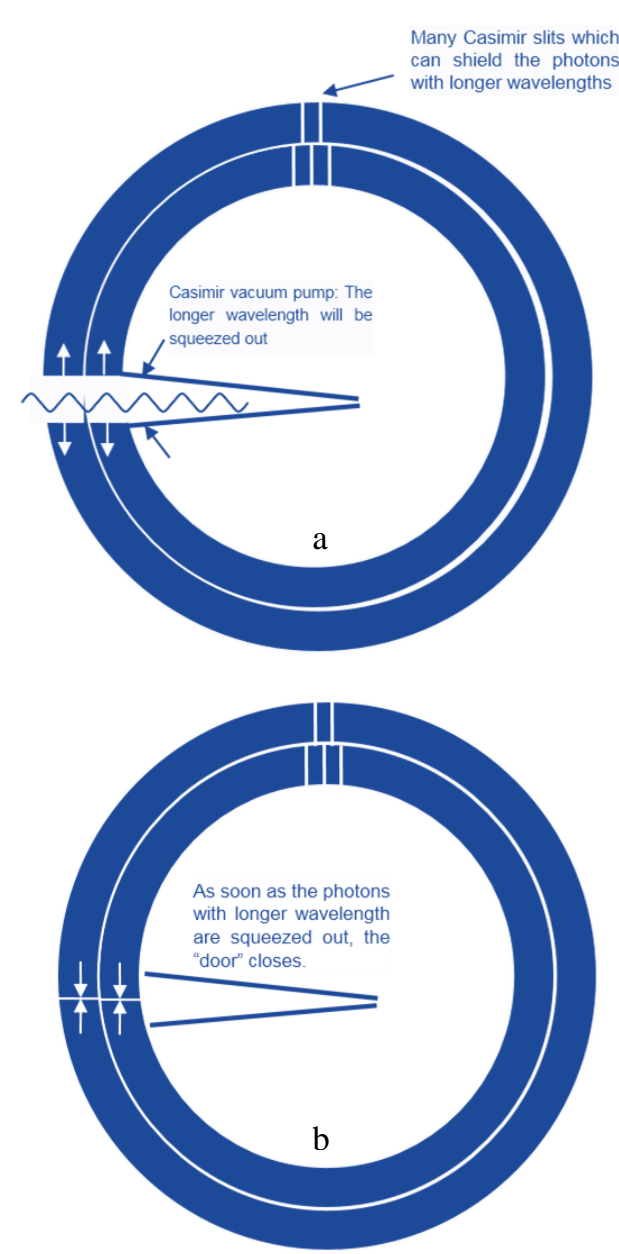

Fig 2. Casimir vacuum pump: The "modern Magdeburg hemispheres" consisting of the hollow spheres with many Casimir slits in the shells, which can shield the photons with longer wavelength. Two Casimir plates are installed in the inner hollow space, which can squeeze the inner photons with longer wavelength out by moving these plates. There are some doors in the shells. As soon as the longer waves are squeezed out, the doors will close.

In the space with Casimir vacuum, one can do the experiment for getting the new absolute zero temperature. One can put $n$ gas molecules into the Casimir vacuum and test the parameters of Charles's law. The Charles's law in Casimir vacuum is

\section{REFERENCES}

Barton, G. (1990). Faster-than-c light between parallel mirrors The Scharnhorst effect rederived. Physics Letters $B, 237(3-4), 559-562$.

Bercegol, H., \& Lehoucq, R. (2015). Vacuum friction on a rotating pair of atoms. Physical review letters, 115(9), 090402.

Chown, M. (1990). Can photons travel'faster than light'? New Sci., 32.

Fischer, J., Gerasimov, S., Hill, K., Machin, G., Moldover, M., Pitre, L., . Ugur, H. (2007). Preparative steps towards the new definition of the kelvin in terms of the Boltzmann

$$
V_{T}=V_{0}\left(1+\frac{T}{\alpha}\right) \quad(\alpha>273.15)
$$

Therefore, the absolute zero in Casimir vacuum is lower than the ordinary one.

\section{CONCLUSION}

Casimir vacuum has different vacuum structure. The energy density in Casimir vacuum is lower than ordinary one. Therefore, the change of vacuum structure will result in change of the kinetic energy of gas molecules. In this paper, the kinetic energy of gas molecules in Casimir vacuum proved to be reduced in two aspects:

1) The number of wave modes in Casimir vacuum is less than that in ordinary vacuum. Therefore, the Rontgen term (a negative energy dissipation term) in Casimir vacuum is smaller than that in ordinary vacuum, which results in reducing the kinetic energy of gas molecules.

2) The kinetic energy of gas molecules can be divided into two parts, $E_{\text {kin-sys }}=E_{\text {self }}+E_{v a c}$. Because of the negative value of $E_{v a c}$, the kinetic energy of gas molecules $E_{\text {kin-sys }}$ is less than that of ordinary vacuum. According equation $\bar{E}_{k i n}=$ $(3 / 2) k_{B} T$, the lower value of $E_{k i n}$ must result in the lower value of $T$ or $k_{B}$, this is just the new absolute zero temperature. Casimir vacuum-the kind of "modern Magdeburg hemispheres" can be used for utilization of the "zero-point energy." This is just as the air vacuum pump can utilize the air energy. If the new absolute zero is found, the "zero-point energy" could be used.

However, what we are worried about is if it violates the law of conservation of energy. The answer is no. Because the "zeropoint energy" is just like the lake on the top of mountain; the water in the lake has high potential energy, but if there is no altitude difference, this energy cannot be used. We need to dig a very deep hole to use this water's potential energy. As we know, we still need energy to dig a deep hole. Meanwhile, the vacuum energy is a hopeful candidate for "Dark Energy." The relation between the "new absolute zero" and the "Dark Energy" is an interesting topic for further investigation.

constant. International journal of thermophysics, 28(6), 1753-1765.

Scharnhorst, K. (1990). On propagation of light in the vacuum between plates. Physics Letters B, 236(3), 354359.

Scharnhorst, K. (1998). The velocities of light in modified QED vacua. Annalen der physik, 7(7-8), 700-709.

Sonnleitner, M., Trautmann, N., \& Barnett, S. M. (2017). Will a decaying atom feel a friction force? Physical review letters, $118(5), 053601$.

Wilkens, M. (1994). Significance of Röntgen current in quantum optics: Spontaneous emission of moving atoms. Physical Review A, 49(1), 570. 